\title{
Matrix solution for the wall impedance of infinitely long multilayer circular beam tubes
}

\begin{abstract}
H. Hahn
Collider-Accelerator Department, Brookhaven National Laboratory, Upton, New York 11973-5000, USA (Received 16 September 2008; revised manuscript received 9 August 2009; published 21 January 2010)

The coupling impedance of beam tubes is a long-standing important topic for particle accelerators that many authors have addressed. The present study was initiated in view of a specific problem, but its novel approach is broadly applicable to the longitudinal and transverse coupling impedances of coated beam tubes or multilayer tubes. The matrix method presented here derives the wall impedance by treating the radial wave propagation of the beam-excited electromagnetic fields in full analogy to longitudinal transmission lines. Starting from the Maxwell equations, the radially transverse magnetic field components are described for monopole and dipole modes by a $2 \times 2$ matrix. Assuming isotropic material properties within one layer, the transverse field components at the inner boundary of a layer uniquely are determined by matrix transfer of the field components at its outer boundary. By imposing power-flow constraints on the matrix, wave impedance mapping and field matching between layers is enforced and replaced by matrix multiplication. The longitudinal and transverse coupling impedances are derived from the wall impedance at the innermost boundary, and the different procedures for its determination are discussed. The matrix method is demonstrated via selected yet representative examples of the welldocumented cases of a stainless-steel tube, and of a graphite collimator.
\end{abstract}

DOI: 10.1103/PhysRevSTAB.13.012002

PACS numbers: $41.20 . \mathrm{Jb}$

\section{INTRODUCTION}

Many authors have addressed the coupling impedance of beam tubes, a long-standing topic of importance for particle accelerators. Thus, Neil and Sessler [1] analyzed the well-known longitudinal, while Laslett, Neil, and Sessler [2] treated the transverse resistive wall impedance of an infinitely thick beam tube. Of more immediate relevance is the seminal paper by Zotter, which gives the impedance of beam tubes of various materials with a finite wall thickness [3]. The solutions for multilayer structures typically are based on an algorithm involving field matching at the boundary layers, and sequential matching of radial wave impedances [4]. Although, in principle, this method allows many layers, the numerical implementation becomes increasingly complex and can be simplified by the codified use of the matrix method presented here. While studying the coated ceramic beam tube in the Relativistic Heavy Ion Collider (RHIC) injection kicker, Hahn noticed the analogy of the radial with axial wave transmission, and developed the concept wherein the matching of the sequential wave impedance is replaced with multiplication of the appropriate matrices relating the electric and magnetic field components in each layer [5]. Independently, but without reference to transmission lines, Lambertson applied matrices to a double metal layer [6]. Using the theory of transmission lines, Vos derived expressions for the longitudinal impedance of multilayer vacuum chambers, but without using matrices [7]. Recently, similar methods were presented as fields transformation matrix formalism, again without taking advantage of transmission-line concepts [8].
The similarity between certain nonuniform structures, such as the multilayer beam tube considered here, with uniform transmission lines was discussed by the researchers at the Massachusetts Institute of Technology Radiation Laboratory. Marcuvitz already pointed out that nonuniform structures may be computed by straightforward engineering methods involving only impedance calculations based on the transmission-line equivalents of these systems [9]. In this class of nonuniform structures, radial transmission is possible for the TM and TE modes separately, without requiring coupling. In the same vein, solutions for the transmission lines imply matrix multiplication for connecting of separate line sections. The transmission-line formalism is central to the present paper, but is done without substituting voltage and currents for the electromagnetic fields.

The work for this paper that started at Brookhaven over a decade ago was continued with the research on the spallation neutron source abort kicker [10]; however, the unpublished internal notes did not lead to a formal presentation. The recent problem of designing a coating for a ceramic beam tube forced the restart of the work, and the matrix method was applied to the actual case of a multilayer ferrite higher order mode absorber in the superconducting electron gun for the Energy Recovery Linac (ERL) at the Relativistic Heavy Ion Collider (RHIC) [11]. The longitudinal impedance part of the matrix method was presented in a poster at the 2009 Particle Accelerator Conference [12].

During this period, considerable progress was made at other laboratories in understanding the transverse coupling impedance. In addition to the standard references on im- 
pedance problems $[4,13]$, this paper rests on and has profited from the various publications at GSI [14], FNAL [15], DESY $[8,16]$, and primarily the experimental and theoretical work at CERN for the LHC collimator [17-19]. The analytic treatments of both the transverse and longitudinal beam tube impedances suffered from a tenuous agreement with experimental data in the low-frequency regime; this issue was addressed successfully in a recent comprehensive paper [20], although, seemingly, further work may be needed.

The goal for this paper was to develop a formally simpler method to find the longitudinal and transverse impedance of infinitely long multilayer circular beam tubes based on a rigorous model valid at all frequencies and beam energies, and to transfer the actual numerical work to the Wolfram MATHEMATICA program. Even so, the required computer programming and execution time can grow significantly, and the numerical examples chosen are for the ultrarelativistic limit only. Furthermore, any finite length effects are not covered and are ignored $[10,21]$.

This paper is organized as follows. Section II establishes the notation used and tabulates the monopole and dipole electromagnetic field components of TE and TM modes that are needed to formulate the longitudinal and transverse impedances. The time-harmonic field components, written for a circular cylindrical layer, are obtained from the homogeneous Helmholtz wave equation with the appropriate boundary conditions but independently of any driving current.

In Sec. III the total coupling impedance seen by the beam as generated by a monopole or dipole tubular driving current is derived by considering the impact of the wall impedance at the innermost beam tube wall. This analytical treatment is characterized by strictly separating the contribution to the beam coupling impedance from the space charge and the contribution from the beam tube wall impedance. As to the terminology used later on and following Ref. [20], the total coupling impedance affecting the beam is obtained as the sum of the (incoherent) spacecharge impedance plus the resistive wall impedance, thus avoiding the traditional use of "resistive wall impedance" for the total beam impedance. Note that the matrix method applies strictly only to the derivation of the wall (surface) impedance. The Panofsky-Wenzel theorem for the dipole modes imposes additional constraints on the total coupling impedance [22], however in the select case of the beam tube geometry considered here, the wall impedance can be obtained by satisfying a single condition.

In the subsequent Sec. IV, the concept of radial wave transmission is fully developed into the matrix method for multilayer beam tubes. The matrix elements are defined, relating the tangential field components at the outer and the inner boundary of a layer. Continuity of radial power flow is assured by appropriate constraints on the matrix describ- ing each layer. It must be emphasized that the sequential field-matching algorithm and the matrix multiplication are substantially equivalent and equally valid, but it is also noted that the application of the matrix method is specially suited to solving multilayer beam problems with the Wolfram MATHEMATICA program.

Section V establishes the correspondence between the transfer of field components across the layer and the mapping of impedances. The paramount importance of the field presentation in the outermost layer to the solution for the wall impedance at the beam tube's inner radius is pointed out, and the special situation of an infinite outer vacuum layer is discussed.

The application of the matrix method is illustrated in Secs. VI and VII by computing and showing the longitudinal- and transverse-wall impedances of the welldocumented PETRA III beam tube [16] and the LHC copper-coated graphite collimator [19]. The paper concludes with a short summary of the pertinent properties of the matrix method.

\section{FIELD PRESENTATION}

The multilayer beam tube is considered as being infinitely long and formed from several concentric circular cylinders of homogenous and isotropic material. Solutions of the Maxwell's equations in the frequency domain, $\exp (j \omega t)$, are found in the absence of free currents separately for each layer from the homogenous scalar wave equation, written in circular cylindrical coordinates, $u=$ $u(r, \varphi, z)[23]$,

$$
\frac{d^{2} u}{d r^{2}}+\frac{d u}{r d r}+\frac{d^{2} u}{r^{2} d \varphi^{2}}-\left(\beta^{-2}-\mu \varepsilon_{S}\right) k^{2} u=0 .
$$

Simplification of the formulas has been achieved by using natural units ( $\left.c=1, \mu_{0}=1, \varepsilon_{0}=1\right)$. Note also the different definition used here for the wave number, $k=\omega$, and the treatment of $\beta$ as a free parameter.

In the subsequent field and matrix equations, a $\beta \leq 1$ refers to the axial beam velocity and implies that the solutions represent waves propagating in the axial direction. A different yet valid set of solutions exists for $\beta=\infty$ which corresponds to an axially uniform beam and to purely radial propagation. In generating the matrix for a layer, the tangential field components on the cylindrical surface are viewed as radially transverse fields defining the impedances as required for the surface impedance at the beam tube inner radius. This approach ensures that the matrix method is exact and equally suited for the analysis of the axial as well as the radial wave propagation model. Choosing the appropriate model is a physics problem rather than a mathematical one. However, unless stated otherwise, this paper retains the more conventional axial formulation for the development of the matrices and the derivation of the beam coupling impedances. A few relevant considerations are made in Sec. V regarding the treat- 
TABLE I. Monopole field components.

\begin{tabular}{lcc}
\hline \hline & $\mathrm{TM}_{I} 0(\kappa r)$ & $\mathrm{TM}_{K} 0(\kappa r)$ \\
\hline$E_{r}$ & $j \frac{k}{\beta \kappa} I_{0}^{\prime}(\kappa r)$ & $j \frac{k}{\beta \kappa} K_{0}^{\prime}(\kappa r)$ \\
$E_{\varphi}$ & 0 & 0 \\
$E_{z}$ & $I_{0}(\kappa r)$ & $K_{0}(\kappa r)$ \\
$H_{r}$ & 0 & 0 \\
$H_{\varphi}$ & $j \frac{k \varepsilon_{S}}{\kappa} I_{0}^{\prime}(\kappa r)$ & $j \frac{k \varepsilon_{S}}{\kappa} K_{0}^{\prime}(\kappa r)$ \\
$H_{z}$ & 0 & 0 \\
\hline \hline
\end{tabular}

ment of the vacuum in the outermost layer and the possible impact on the low-frequency wall impedance.

Solutions of Eq. (1) are obtained as linear combinations of cylinder functions, written here in terms of modified Bessel functions with the argument $(\kappa r)$

$$
\begin{gathered}
u_{\|} \sim \frac{1}{\kappa^{2}}\left\{I_{0}(\kappa r) ; K_{0}(\kappa r)\right\} \exp \left(-\beta^{-1} k z\right) \\
u_{T} \sim \frac{\cos \varphi}{\kappa^{2}}\left\{I_{1}(\kappa r) ; K_{1}(\kappa r)\right\} \exp \left(-\beta^{-1} k z\right) .
\end{gathered}
$$

The radial propagation constant,

$$
\kappa^{2}=\left(\beta^{-2}-\mu \varepsilon_{S}\right) k^{2},
$$

is defined by the material parameters permeability, $\mu=$ $\mu^{\prime}-j \mu^{\prime \prime}$, and permittivity plus conductivity, $\varepsilon_{S}=\varepsilon^{\prime}-$ $j\left(\varepsilon^{\prime \prime}+\sigma / k\right)$. Table I shows explicit expressions for monopole fields and Table II for dipole fields [24].

\section{TOTAL COUPLING IMPEDANCE}

The electromagnetic field excited by a time-harmonic current within a layer of the multilayered beam tube can be represented by linear combinations of elementary functions, found as solutions to the scalar wave equation. To prevent a logarithmic divergence of the result for the spacecharge impedance, the beam can be given a finite transverse size. Solving the case of a tubular beam with radius, $a$, avoids such divergence. The tubular beam of current, $I$, is assumed to travel in the $z$ direction with velocity $\beta$ and has the longitudinal current density [with the timeharmonic factor $\exp (j \omega t)$ suppressed]

$$
i_{z}=\frac{I}{2 \pi a} \delta(r-a) e^{-j \beta^{-1} k z}
$$

or for a dipole current

$$
i_{z}=\frac{I}{\pi a} \cos \varphi \delta(r-a) e^{-j \beta^{-1} k z} .
$$

The foundation for the present study is laid by first deriving the expression for the longitudinal and transverse coupling impedance seen by an axial beam in an evacuated beam tube, $\varepsilon=\mu=1$, with known wall or "surface" impedance $R(b)=-E_{z}(b) / H_{\varphi}(b)$ at the innermost beam tube radius. The beam tube properties are fully defined by its wall impedance which can be found independently of the beam, even in the case for a layered tube. Again, it is noted here that the total coupling impedance consists of two parts, the space charge plus a separate contribution from the beam tube wall impedance. The space charge is obtained by considering the beam tube as a perfect conductor, $R(b)=0$. Space charge is energy dependent as is wall impedance in the axial modal. Although this paper treats the energy dependence rigorously, the simplified results for an ultrarelativistic and filamentary beam also are given.

\section{A. Longitudinal beam impedance}

The electromagnetic monopole fields in the beam tube, generated by the tubular current, with the common timeharmonic factor omitted and noting again that $k=\omega$, are found inside the current tube,

$$
E_{z i}(\eta r)=A I_{0}(\eta r) \quad H_{\varphi i}(\eta r)=j A \beta \gamma I_{1}(\eta r),
$$

and outside of the current tube,

$$
\begin{aligned}
& E_{z o}(\eta r)=A \frac{I_{0}(\eta a)\left[K_{0}(\eta r)+\alpha I_{0}(\eta r)\right]}{K_{0}(\eta a)+\alpha I_{0}(\eta a)} \\
& H_{\varphi o}(\eta r)=j A \beta \gamma \frac{I_{0}(\eta a)\left[-K_{1}(\eta r)+\alpha I_{1}(\eta r)\right]}{K_{0}(\eta a)+\alpha I_{0}(\eta a)},
\end{aligned}
$$

with $\eta=k / \beta \gamma, \beta$ the relativistic velocity factor, $\gamma$ the relativistic mass factor, and $A$ and $\alpha$ free coefficients yet to be determined. Matching of $E_{z}(a)$ is built into the expressions and imposing Ampere's law at $r=a$ leads to

TABLE II. Dipole field components.

\begin{tabular}{lccccc}
\hline \hline & $\mathrm{TM}_{I} 1(\kappa r)$ & $\mathrm{TE}_{I} 1(\kappa r)$ & $\mathrm{TM}_{K} 1(\kappa r)$ & $\mathrm{TE}_{K} 1(\kappa r)$ & \\
\hline$E_{r}$ & $j \frac{k}{\beta \kappa} I_{1}^{\prime}(\kappa r)$ & $j \frac{k \mu}{\kappa^{2} r} I_{1}(\kappa r)$ & $j \frac{k}{\beta \kappa} K_{1}^{\prime}(\kappa r)$ & $j \frac{k \mu}{\kappa^{2} r} K_{1}(\kappa r)$ & $\cos \varphi$ \\
$E_{\varphi}$ & $-j \frac{k}{\beta \kappa^{2} r} I_{1}(\kappa r)$ & $-j \frac{k \mu}{\kappa} I_{1}^{\prime}(\kappa r)$ & $-j \frac{k}{\beta \kappa^{2} r} K_{1}(\kappa r)$ & $-j \frac{k \mu}{\kappa} K_{1}^{\prime}(\kappa r)$ & $\sin \varphi$ \\
$E_{z}$ & $I_{1}(\kappa r)$ & 0 & $K_{1}(\kappa r)$ & 0 & $\cos \varphi$ \\
$H_{r}$ & $j \frac{k \varepsilon_{S}}{\kappa^{2} r} I_{1}(\kappa r)$ & $j \frac{k}{\beta \kappa} I_{1}^{\prime}(\kappa r)$ & $j \frac{k \varepsilon_{S}}{2^{2} r} K_{1}(\kappa r)$ & $j \frac{k}{\beta \kappa} K_{1}^{\prime}(\kappa r)$ & $\sin \varphi$ \\
$H_{\varphi}$ & $j \frac{k \varepsilon_{S}}{\kappa} I_{1}^{\prime}(\kappa r)$ & $j \frac{k}{\beta \kappa^{2} r} I_{1}(\kappa r)$ & $j \frac{k \varepsilon_{S}}{\kappa} K_{1}^{\prime}(\kappa r)$ & $j \frac{k}{\beta \kappa^{2} r} K_{1}(\kappa r)$ & $\cos \varphi$ \\
$H_{z}$ & 0 & $I_{1}(\kappa r)$ & 0 & $K_{1}(\kappa r)$ & $\sin \varphi$ \\
\hline \hline
\end{tabular}




$$
A=j \frac{k}{2 \pi a(\beta \gamma)^{2}}\left[K_{0}(\eta a)+\alpha I_{0}(\eta a)\right] .
$$

Matching the fields to the wall impedance value, $R(b)=$ $-E_{z}(b) / H_{\varphi}(b)$ yields

$$
\alpha=\frac{j \beta \gamma R(b) K_{1}(\eta b)-K_{0}(\eta b)}{I_{0}(\eta b)+j \beta \gamma R(b) I_{1}(\eta \kappa b)} .
$$

Finally, the longitudinal coupling impedance per unit length follows as

$$
\begin{aligned}
Z_{\|}= & -\frac{E_{z}(a)}{I} \\
= & -j \frac{k I_{0}(\eta a)}{2 \pi(\beta \gamma)^{2}} \\
& \times\left\{K_{0}(\eta a)-\frac{I_{0}(\eta a)\left[K_{0}(\eta b)-j R(b) \beta \gamma K_{1}(\eta b)\right]}{I_{0}(\eta b)+j R(b) \beta \gamma I_{1}(\eta b)}\right\} .
\end{aligned}
$$

The total result can be separated into the space charge plus the resistive wall impedance,

$$
\begin{aligned}
Z_{\|}= & -j \frac{k}{2 \pi(\beta \gamma)^{2}} \frac{I_{0}(\eta a)}{I_{0}(\eta b)} \\
& \times\left[I_{0}(\eta b) K_{0}(\eta a)-I_{0}(\eta a) K_{0}(\eta b)\right] \\
& +\frac{R(b)}{2 \pi b} \frac{I_{0}^{2}(\eta a)}{I_{0}^{2}(\eta b)+j R(b) \beta \gamma I_{0}(\eta b) I_{1}(\eta b)} .
\end{aligned}
$$

The total impedance is simplified for the ultrarelativistic limit, $\eta \rightarrow 0$, and one step further for a filamentary beam into the expression

$$
\begin{aligned}
\lim _{\beta \gamma \rightarrow \infty} Z_{\|} \approx & -j \frac{k}{2 \pi(\beta \gamma)^{2}} \ln \left(\frac{b}{a}\right)+\frac{R(b)}{2 \pi b}\left(\frac{1}{1+j k b R(b) / 2}\right) \\
& +O\left(\frac{1}{(\beta \gamma)^{2}}\right) .
\end{aligned}
$$

The ultrarelativistic part is consistent with Chao's general solution, Eq. (2.75) in Ref. [25], and reduces in the low frequency limit to

$$
\lim _{\beta \gamma \rightarrow \infty} Z_{\|} \approx \frac{R(b)}{2 \pi b} .
$$

\section{B. Transverse Beam impedance}

The transverse beam tube impedance seen by the dipole current defined in Eq. (6) is obtained from the longitudinal electric field component at the tubular current $r=a$ according to [13]:

$$
Z_{\perp}=-\frac{2}{k a^{2}} \frac{E_{z i}(a)}{I} .
$$

The dipole generated field components are found to be inside of the tubular current,

$$
\begin{aligned}
& E_{z i}(r)=A_{E i} I_{1}(\kappa r) \\
& H_{\varphi i}(r)=j \frac{k}{\kappa}\left\{A_{E i} \varepsilon I_{1}^{\prime}(\kappa r)+A_{H i} \frac{I_{1}(\kappa r)}{\beta \kappa r}\right\} \\
& E_{\varphi i}(r)=-j \frac{k}{\kappa}\left\{A_{E i} \frac{I_{1}(\kappa r)}{\beta \kappa r}+A_{H i} \mu I_{1}^{\prime}(\kappa r)\right\} \\
& H_{z i}(r)=A_{H i} I_{1}(\kappa r),
\end{aligned}
$$

and the fields outside of the tubular current are

$$
\begin{aligned}
E_{z o}(r)= & A_{E} I_{1}(\kappa r)+B_{E} K_{1}(\kappa r) \\
H_{\varphi o}(r)= & j \frac{k}{\kappa}\left\{A_{E} \varepsilon I_{1}^{\prime}(\kappa r)+A_{H} \frac{I_{1}(\kappa r)}{\beta \kappa r}+B_{E} \varepsilon K_{1}^{\prime}(\kappa r)\right. \\
& \left.+B_{H} \frac{K_{1}(\kappa r)}{\beta \kappa r}\right\} \\
E_{\varphi o}(r)= & -j \frac{k}{\kappa}\left\{A_{E} \frac{I_{1}(\kappa r)}{\beta \kappa r}+A_{H} \mu I_{1}^{\prime}(\kappa r)\right. \\
& \left.+B_{E} \frac{K_{1}(\kappa r)}{\beta \kappa r}+B_{H} \mu K_{1}^{\prime}(\kappa r)\right\} \\
H_{z o}(r)= & A_{H} I_{1}(\kappa r)+B_{H} K_{1}(\kappa r) .
\end{aligned}
$$

Field matching of the fields at the tubular beam requires

$$
\begin{gathered}
E_{z i}(a)=E_{z o}(a) \quad E_{\varphi i}(a)=E_{\varphi o}(a) \\
H_{z i}(a)=H_{z o}(a) \quad H_{\varphi o}(a)=H_{\varphi i}(a)+\frac{I}{\pi a} .
\end{gathered}
$$

Imposing the (resistive) wall impedance leads to additional constraints on the free coefficients

$$
E_{z o}(b)=-R_{z} H_{\varphi o}(b) \quad E_{\varphi o}(b)=R_{\varphi} H_{z o}(b) .
$$

The induced dipole fields with vacuum inside the beam tube are obtained by substituting $\kappa^{2}$ with $\eta^{2}=\left(\beta^{-2}-\right.$ $\mu \varepsilon) k^{2}$ leading to the relativistic $\eta=k / \beta \gamma$. The free coefficients are readily determined by the Wolfram MATHEMATICA program. After simple manipulations, the expression for the impedance determining coefficient $A_{E i}$ is found as

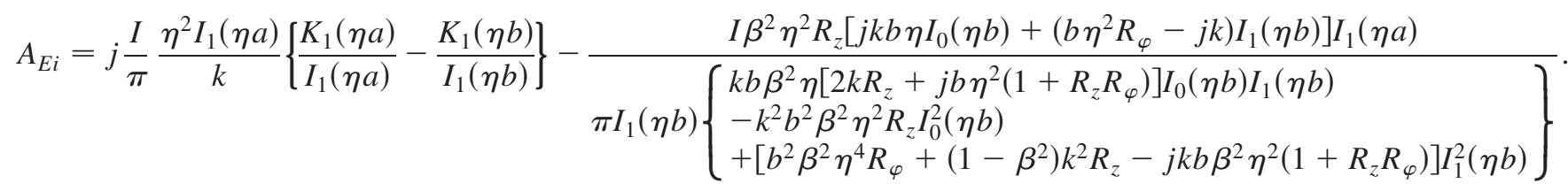


The expression is written so that the first term represents the space-charge term and the second the resistive wall impedance. The transverse impedance now follows from the longitudinal electric field component at the tubular current according to Eq. (15).

The expression for the transverse coupling impedance involves both wall impedances, $R_{z}$ and $R_{\varphi}$, whereas the longitudinal coupling impedance is fully determined by the $R_{z}$ wall impedance alone. Although $R_{z}$ is defined equally for monopole and dipole modes it is evaluated differently. The Panofsky-Wenzel theorem predicts that the transverse coupling impedance follows from the longitudinal one, implying that it, too, is only determined by $R_{z}$ [22]. Verifying this condition in general is lengthy and beyond the scope of this paper. However, the coupling impedance expression for the ultrarelativistic case is found by taking first $\beta \rightarrow 1$ and then $\eta=k / \beta \gamma \rightarrow 0$, leading to

$$
Z_{T}=\frac{2}{\pi b^{2}} \frac{R_{z}}{k b\left(1+j k b R_{z} / 2+R_{z} R_{\varphi}\right)} .
$$

Imposing the single condition, $R_{\varphi}=0$, at the beam tube wall satisfies the Panofsky-Wenzel theorem, yielding the formula for the transverse coupling impedance,

$$
Z_{T}=\frac{2}{\pi b^{2}} \frac{R_{z}}{k b\left(1+j k b R_{z} / 2\right)} \approx \frac{2}{\pi b^{2}} Z_{\|},
$$

and at the same time provides a considerable simplification of the field solution for layered beam tubes. In fact, together with the Marcuvitz discussion of radial transmission lines [9], it negates the need for transverse electric modes and allows a $2 \times 2$ matrix treatment of the transverse-wall impedance. Equation (22), connecting the transverse and longitudinal coupling impedances, is a frequently used relation, which is valid in the classic resistive thick wall approximation, but only qualitative in the general case.

\section{MATRIX METHOD}

In most previous publications, the coupling impedance solution for multilayer structures was obtained with Zotter's algorithm involving field matching at the boundary layers and sequential mapping of radial wave impedances [4]. This paper presents the alternative matrix method wherein in full analogy to the treatment of longitudinal transmission lines the field matching is replaced by the multiplication of radial transfer matrices. In this method, the fields at the innermost layer, $r=b$, are determined via an overall matrix by mapping the radial wave impedance at the last beam tube layer, $R_{z}\left(r_{o}\right)$, which is at the inner layer of an unbound, infinitely extended outermost layer. Both methods are mathematically equivalent and exact for a model with fixed geometry and material properties.

The wall impedance of a multilayer structure is obtained by properly matching the tangential field components at the cylinder boundaries with the matching best done by multiplication of the radial transfer matrices for each layer. Thus, the overall matrix of a sequence of radially spaced cylinders is represented as the sequential product of all individual matrices, starting from the most inward radius, $b$, to the outermost radius $r_{o}$,

$$
M\left(b, r_{o}\right)=M^{I}\left(b, r_{1}\right) \cdot M^{I I}\left(r_{1}, r_{2}\right) \cdots M^{I I I}\left(r_{o-1}, r_{o}\right) .
$$

In contrast to engineering transmission-line theory that relates voltages and currents, the matrix here relates the electric and magnet fields. Rigorous expressions of matrices defining the monopole and dipole fields are collected in this section for typical wall materials. Expressions for the matrix elements are given in terms of Bessel functions or modified Bessel functions, and the choice depends only on which asymptotic expansion is better suited to a particular material parameter range.

\section{A. Monopole matrices}

The monopole matrix for a layer, ranging from one radius $r$ within the layer inclusive of the inner boundary $r_{i}$ to the reference radius at the outer layer boundary $r_{0}$, is defined in the general form

$$
\begin{aligned}
\left(\begin{array}{c}
E_{z}(r) \\
H_{\varphi}(r)
\end{array}\right) & =M\left(r, r_{0}\right)\left(\begin{array}{c}
E_{z}\left(r_{0}\right) \\
H_{\varphi}\left(r_{0}\right)
\end{array}\right) \\
& =\left[\begin{array}{cc}
m_{e e}\left(r, r_{0}\right) & m_{e h}\left(r, r_{0}\right) \\
m_{h e}\left(r, r_{0}\right) & m_{h h}\left(r, r_{0}\right)
\end{array}\right]\left(\begin{array}{c}
E_{z}\left(r_{0}\right) \\
H_{\varphi}\left(r_{0}\right)
\end{array}\right)
\end{aligned}
$$

with the elements satisfying certain constraints to achieve power flow in addition to field component matching. At the reference radius, $r=r_{0}$, the matrix is required to read as

$$
M\left(r_{0}, r_{0}\right)=\left[\begin{array}{ll}
1 & 0 \\
0 & 1
\end{array}\right] .
$$

The matrix (24) relates the electric and magnet fields at all radial positions, $b<r<r_{o}$, while imposing the constraint of power flow continuity via the Wronskian with the requirement that

$$
\operatorname{det} M\left(r, r_{o}\right)=r_{o} / r
$$

Broad coverage for most materials, including lossy metals, is found with modified Bessel functions of order zero and order one and the argument $(\kappa r)$

$$
\begin{aligned}
& m_{e e}\left(r, r_{0}\right)=\kappa r_{0}\left[K_{0}(\kappa r) I_{1}\left(\kappa r_{0}\right)+I_{0}(\kappa r) K_{1}\left(\kappa r_{0}\right)\right] \\
& m_{e h}\left(r, r_{0}\right)=j \frac{\kappa^{2} r_{0}}{\varepsilon_{S} k}\left[K_{0}(\kappa r) I_{0}\left(\kappa r_{0}\right)-I_{0}(\kappa r) K_{0}\left(\kappa r_{0}\right)\right] \\
& m_{h e}\left(r, r_{0}\right)=-j \varepsilon_{S} k r_{0}\left[K_{1}(\kappa r) I_{1}\left(\kappa r_{0}\right)-I_{1}(\kappa r) K_{1}\left(\kappa r_{0}\right)\right] \\
& m_{h h}\left(r, r_{0}\right)=\kappa r_{o}\left[K_{1}(\kappa r) I_{0}\left(\kappa r_{0}\right)+I_{1}(\kappa r) K_{0}\left(\kappa r_{0}\right)\right],
\end{aligned}
$$

where the radial propagation constant in each layer is determined by Eq. (4), $\kappa^{2}=\left(\beta^{-2}-\mu \varepsilon_{S}\right) k^{2}$, with the 
material properties for permittivity plus conductivity, $\varepsilon_{S}=$ $\varepsilon^{\prime}-j\left(\varepsilon^{\prime \prime}+\sigma / k\right)$, and permeability, $\mu=\mu^{\prime}-j \mu^{\prime \prime}$.

\section{Matrix of high-conductivity metal}

The impedance of a metallic beam tube represents the classic example for the resistive wall instability. In highconductivity metals $\varepsilon_{S} \approx-j \sigma / k$ leading to $\kappa \rightarrow \chi=$ $\sqrt{j \mu \sigma k}$, suggesting the use of Hankel functions instead; however, in the Wolfram MATHEMATICA environment they bring no simplification beyond the asymptotic expansions of modified Bessel functions for large arguments frequently found in the literature. Although intended primarily for use at high frequencies, the monopole matrix for a high-conductivity material can take the simpler form,

$$
\left(\begin{array}{c}
E_{z}(r) \\
H_{\varphi}(r)
\end{array}\right)=\left[\begin{array}{cc}
C_{0} & \underline{\chi} S_{0} \\
\frac{\sigma}{\chi} C_{0}^{\prime} & S_{0}^{\prime}
\end{array}\right]\left(\begin{array}{c}
E_{z}\left(r_{0}\right) \\
H_{\varphi}\left(r_{0}\right)
\end{array}\right)
$$

with $C_{0}$ and $S_{0}$ given exactly by the modified Bessel function combinations, or by their high frequency hyperbolic approximations,

$$
\begin{aligned}
C_{0} & =\chi r_{0}\left[K_{0}(\chi r) I_{1}\left(\chi r_{0}\right)+I_{0}(\chi r) K_{1}\left(\chi r_{0}\right)\right] \\
& \approx \sqrt{\frac{r_{0}}{r}} \cosh \chi\left(r-r_{0}\right) \\
C_{0}^{\prime} & =\chi r_{0}\left[-K_{1}(\chi r) I_{1}\left(\chi r_{0}\right)+I_{1}(\chi r) K_{1}\left(\chi r_{0}\right)\right] \\
& \approx \sqrt{\frac{r_{0}}{r}} \sinh \chi\left(r-r_{0}\right) \\
S_{0} & =\chi r_{0}\left[-K_{0}(\chi r) I_{0}\left(\chi r_{0}\right)+I_{0}(\chi r) K_{0}\left(\chi r_{0}\right)\right] \\
& \approx \sqrt{\frac{r_{0}}{r}} \sinh \chi\left(r-r_{0}\right) \\
S_{0}^{\prime} & =\chi r_{0}\left[K_{1}(\chi r) I_{0}\left(\chi r_{0}\right)+I_{1}(\chi r) K_{0}\left(\chi r_{0}\right)\right] \\
& \approx \sqrt{\frac{r_{0}}{r}} \cosh \chi\left(r-r_{0}\right) .
\end{aligned}
$$

\section{Metallic coating}

The general expression for the case of high-conductivity metal coating was given above, but as an additional exercise the matrix for the thin-layer approximation is given here. Taylor expansion to the second order in $\Delta=r_{o}-r_{i}$ of the full matrix elements leads to

$$
\begin{gathered}
m_{e e} \approx 1+j \frac{1}{2} \sigma k \Delta^{2} \quad m_{e h} \approx-j k \Delta\left(1+\frac{1}{2} \frac{k \Delta^{2}}{r_{i}}\right) \\
m_{h e} \approx-\sigma \Delta\left(1+\frac{1}{2} \frac{k \Delta^{2}}{r_{i}}\right) \quad m_{h h} \approx 1+\frac{\Delta}{r_{i}}+j \frac{1}{2} \sigma k \Delta^{2} .
\end{gathered}
$$

\section{Ceramic beam tube}

The matrix of a ceramic tube is described with Bessel functions of order zero and one and the wave number, $\nu=$ $k \sqrt{\mu \varepsilon_{S}-\beta^{-2}}$,

$$
\begin{aligned}
& m_{e e}\left(r, r_{0}\right)=\frac{\pi \nu r_{0}}{2}\left[Y_{0}(\nu r) J_{1}\left(\nu r_{0}\right)-J_{0}(\nu r) Y_{1}\left(\nu r_{0}\right)\right] \\
& m_{e h}\left(r, r_{0}\right)=-j \frac{\pi \nu^{2} r_{0}}{2 \varepsilon_{S} k}\left[Y_{0}\left(\nu r_{0}\right) J_{0}(\nu r)-J_{0}\left(\nu r_{0}\right) Y_{0}(\nu r)\right] \\
& m_{h e}\left(r, r_{0}\right)=j \frac{\pi \varepsilon_{S} k r_{0}}{2}\left[Y_{1}(\nu r) J_{1}\left(\nu r_{0}\right)-J_{1}(\nu r) Y_{1}\left(\nu r_{0}\right)\right] \\
& m_{h h}\left(r, r_{0}\right)=\frac{\pi \nu r_{0}}{2}\left[Y_{0}\left(\nu r_{0}\right) J_{1}(\nu r)-J_{0}\left(\nu r_{0}\right) Y_{1}(\nu r)\right] .
\end{aligned}
$$

\section{Matrix of air (vacuum) between two layers}

Following the usual practice of ignoring the small nonzero electric susceptibility, $\left(\varepsilon^{\prime}-1\right) \approx 6 \times 10^{-4}$ for the sake of simplicity, air is treated as vacuum. A practical matrix for vacuum between two layers, extending from $r$ to $r_{0}$, follows in the ultrarelativistic approximation, $\kappa \rightarrow \eta \simeq$ $k / \beta \gamma \rightarrow 0$, as

$$
\begin{gathered}
m_{e e}\left(r, r_{0}\right) \rightarrow 1 \quad m_{e h}\left(r, r_{0}\right) \rightarrow 0 \\
m_{h e}\left(r, r_{0}\right) \rightarrow j \frac{k r}{2}\left[1-\frac{r_{0}^{2}}{r^{2}}\right] \quad m_{h h}\left(r, r_{0}\right) \rightarrow \frac{r_{0}}{r} .
\end{gathered}
$$

\section{B. Dipole matrices}

The dipole wave transmission properties of each layer in general are defined by a $4 \times 4$ matrix. However, the geometry of the multilayer beam tube is a perfect example for radial transmission lines, wherein the requirement of enforcing $R_{\varphi}=0$ simplifies finding the coupling impedance with only the TM modes, and thus a $2 \times 2$ matrix is adequate.

In a straightforward extension, one can define the appropriate dipole matrix in the general form,

$$
\left(\begin{array}{l}
E_{z}(r) \\
H_{\varphi}(r)
\end{array}\right)=\left[\begin{array}{ll}
m_{\Theta e e}\left(r, r_{0}\right) & m_{\Theta e h}\left(r, r_{0}\right) \\
m_{\Theta h e}\left(r, r_{0}\right) & m_{\Theta h h}\left(r, r_{0}\right)
\end{array}\right]\left(\begin{array}{l}
E_{z}\left(r_{0}\right) \\
H_{\varphi}\left(r_{0}\right)
\end{array}\right) .
$$

The dipole solution of the scalar wave equation for a broad range of materials requires combining the modified Bessel functions with the expressions from Table II, leading to

$$
\left(\begin{array}{l}
E_{z}(r) \\
H_{\varphi}(r)
\end{array}\right)=\left[\begin{array}{cc}
C_{1} & -j \frac{\kappa}{k \varepsilon_{S}} S_{1} \\
j \frac{k \varepsilon_{S}}{\kappa} C^{\prime} & S^{\prime}
\end{array}\right]\left(\begin{array}{l}
E_{z}\left(r_{0}\right) \\
H_{\varphi}\left(r_{0}\right)
\end{array}\right) .
$$

The radial functions $C_{1}$ and $S_{1}$ in Eq. (34) are listed here, together with their usual approximations although the accuracy of the hyperbolic approximations at low frequencies is questionable, 


$$
\begin{aligned}
C_{1} & =\kappa r_{0}\left\{K_{1}(\kappa r) I_{1}^{\prime}\left(\kappa r_{0}\right)-I_{1}(\kappa r) K_{1}^{\prime}\left(\kappa r_{0}\right)\right\} \\
& \approx \sqrt{r_{0} / r} \cosh \kappa\left(r-r_{0}\right) \\
C_{1}^{\prime} & =\kappa r_{0}\left\{K_{1}^{\prime}(\kappa r) I_{1}^{\prime}\left(\kappa r_{0}\right)-I_{1}^{\prime}(\kappa r) K_{1}^{\prime}\left(\kappa r_{0}\right)\right\} \\
& \approx \sqrt{r_{0} / r} \sinh \kappa\left(r-r_{0}\right) \\
S_{1} & =\kappa r_{0}\left\{I_{1}(\kappa r) K_{1}\left(\kappa r_{0}\right)-K_{1}(\kappa r) I_{1}\left(\kappa r_{0}\right)\right\} \\
& \approx \sqrt{r_{0} / r} \sinh \kappa\left(r-r_{0}\right) \\
S_{1}^{\prime} & =\kappa r_{0}\left\{I_{1}^{\prime}(\kappa r) K_{1}\left(\kappa r_{0}\right)-K_{1}^{\prime}(\kappa r) I_{1}\left(\kappa r_{0}\right)\right\} \\
& \approx \sqrt{r_{0} / r} \cosh \kappa\left(r-r_{0}\right) .
\end{aligned}
$$

At the reference radius $r=r_{0}$, the matrix must read as

$$
M_{\Theta}\left(r_{0}, r_{0}\right)=\left[\begin{array}{ll}
1 & 0 \\
0 & 1
\end{array}\right]
$$

and continuity of the radial power flow requires via the Wronskian that

$$
\operatorname{det} M_{\Theta}=r_{0} / r .
$$

It is noted that the matrix representation of layers in Eqs. (24) and (33) is a general one and not tied to a specific field analysis, be it the axial or radial propagation model.

\section{FIELD MAPPING}

The electromagnetic fields, and concomitantly the impedances, at the outer radius of a layer are "mapped" by the matrix to the inside radius. Mapping across a single layer can be generalized to the case of a multilayer beam tube. In the matrix $M\left(r, r_{o}\right)$, the radius $r_{o}$ is defined as the outermost beam tube radius if it is terminated by a vacuum, or as the inner radius of the outermost material layer if infinite. In all cases, the properties of the terminating layer, material extending to infinite or to a vacuum, are important and deserve a separate treatment.

\section{A. Impedance mapping}

For simplicity, the infinitely extended outermost layer is assumed to be isotropic and is characterized by its material properties, with $\kappa$ as defined above, leading to the wave impedances for monopole $\mathrm{TM}_{01}$ waves at $r_{o}$,

$$
R_{z}\left(r_{o}\right)=-\frac{E_{z}\left(r_{o}\right)}{H_{\varphi}\left(r_{o}\right)}=j \frac{\kappa}{k \varepsilon_{S}} \frac{K_{0}\left(\kappa r_{o}\right)}{K_{0}^{\prime}\left(\kappa r_{o}\right)} .
$$

The longitudinal wall impedance at the beam tube inner radius, $r_{i}=b$, is found in terms of the matrix formulation to be

$$
R(b)=\frac{M_{e e} R_{z}\left(r_{o}\right)-M_{e h}}{M_{h h}-M_{h e} R_{z}\left(r_{o}\right)} .
$$

In full correspondence, one finds for the $\mathrm{TM}_{11}$ modes

$$
R_{z}\left(r_{o}\right)=j \frac{\kappa}{k \varepsilon_{S}} \frac{K_{1}\left(\kappa r_{o}\right)}{K_{1}^{\prime}\left(\kappa r_{o}\right)}
$$

with the transverse-wall impedance given as

$$
R(b)=\frac{M_{\Theta e e} R_{z}\left(r_{o}\right)-M_{\Theta e h}}{M_{\Theta h h}-M_{\Theta h e} R_{z}\left(r_{o}\right)} .
$$

\section{B. Vacuum as the outermost layer}

A finite thickness, single or multilayer beam tube is necessarily terminated by an infinite vacuum layer. The case of vacuum as the outermost layer requires special treatment, with emphasis on the ultrarelativistic limit. The radial wave number for vacuum with $\varepsilon=\mu=1$ is found as

$$
\kappa \rightarrow \eta=\frac{k}{\beta} \sqrt{1-\beta^{2}}=\frac{k}{\beta \gamma} .
$$

The transverse wave impedances of vacuum for the monopole and dipole modes become

$$
\lim _{\eta \rightarrow 0} R_{z}\left(r_{o}\right) \rightarrow 0 .
$$

Applying these limit values to Eqs. (39) and (41) yields the longitudinal and transverse-wall impedances in the ultrarelativistic limit:

$$
R(b) \approx-\frac{M_{e h}}{M_{h h}}
$$

and

$$
R(b) \approx-\frac{M_{\Theta e h}}{M_{\Theta h h}} .
$$

The present results were derived by the aid of the matrix method within the assumption of axial transmission, that is, with the phase velocity in the material and the vacuum layer equal to that of the beam. In the final two sections, these solutions, which are exact in the ultrarelativistic limit, are illustrated against well-documented structures.

\section{Radial wave propagation}

According to the standard axial propagation assumption (the axial model), the axial electric field in the terminating vacuum layer vanishes in the ultrarelativistic limit and due to field matching forces the electric field to zero at the boundary in the last material layer, seemingly contradicting the skin effect analysis for a tubular conductor [26]. A mathematically valid solution yielding a finite vacuum impedance is found in the radial wave propagation concept (the radial model) which is applied to radiation problems [27]. The translation of this model into the matrix frame work is done by imposing the phase velocity $\beta=\infty$ in the wave equation, Eq. (1), and in the matrix expressions for all material layers. The typical material parameters render the matrix expressions insensitive to $\beta$ so that the results 
derived with the standard axial model and the here propounded radial one are interchangeable at high frequencies and any differences appear mostly at the extreme low frequencies.

The radial wave solutions for monopole fields in the outermost layer, if a vacuum, were derived in contrast to axial waves as circularly symmetric cylindrical radial waves from

$$
u_{0}=H_{0}^{(2)}(k r) \approx \sqrt{\frac{2}{\pi k r}} \exp \left\{-j\left(k r-\frac{\pi}{4}\right)\right\} ; \quad(k r \gg 1) .
$$

Over the cylindrical surfaces of constant $r$, the tangential $E_{z}$ and $H_{\varphi}$ fields are perpendicular to each other at every point, are normal to the direction of propagation, and thus are creating a radial Poynting vector. The results for the monopole impedance of an unbound vacuum layer is given by the ratio $R_{z}=-E_{z} / H_{\varphi}$ as

$$
R_{z}(r)=j \frac{H_{0}^{(2)}(k r)}{H_{1}^{(2)}(k r)} .
$$

Figure 1 shows the dependence of $R_{z}$ on the radial wave number, and as expected the fields approach in the limit of $r \rightarrow \infty$ a plane wave with $R_{z} \sim 1$. The radial model leads to the expression for the longitudinal wall impedance,

$$
R(b)=\frac{M_{e e} R_{z}\left(r_{o}\right)-M_{e h}}{M_{h h}-M_{h e} R_{z}\left(r_{o}\right)},
$$

that in contrast to the axial model is energy independent and thus without simplification for the ultrarelativistic case. Note, however, that the energy dependence of the total coupling impedance enters through the space-charge term.

The dipole impedance of an unbound vacuum layer is found to be

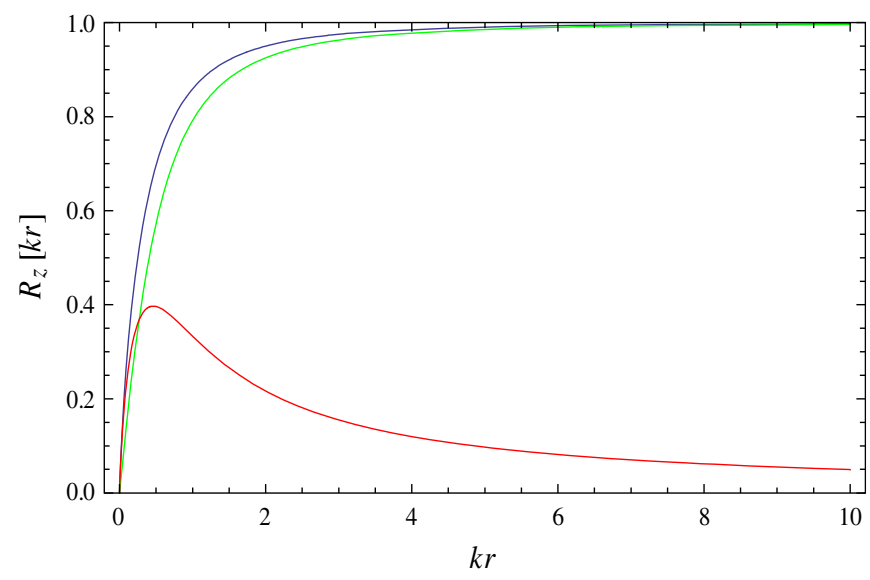

FIG. 1. Absolute value of the radial wave impedance $R_{z}$ versus normalized frequency $k r$ in blue, with real part in green and imaginary part in red.

$$
R_{z}(r)=j H_{1}^{(2)}(k r) / \frac{d H_{1}^{(2)}(k r)}{d(k r)}
$$

and correspondingly the dipole wall impedance,

$$
R(b)=\frac{M_{\Theta e e} R_{z}\left(r_{o}\right)-M_{\Theta e h}}{M_{\Theta h h}-M_{\Theta h e} R_{z}\left(r_{o}\right)} .
$$

It is worth recalling that the original analysis of the resistive wall instability was done based on the conventional skin effect without regard to the direction of power flow in the beam tube $[1,2]$. In fact, it might be questioned which treatment of the beam tube wall impedance, axial or radial propagation, although both mathematically correct, represents the best model to physical reality. The burden of proof for adopting the radial model must be provided by applying it to simple structures for which reliable experimental low-frequency data are available, and which can be used in a comparison with computational results based on the standard axial model. As a first attempt, the longitudinal impedance of the standard PETRA III beam tube and the LHC collimator in the ultrarelativistic limit were selected. A general review of the radial wave propagation involving also dipole modes is beyond the scope of this paper, and is left for a future study.

\section{ULTRARELATIVISTIC LONGITUDINAL IMPEDANCE OF METALLIC BEAM TUBES}

\section{A. Metallic beam tube radially unbound}

The coupling impedance of an unbound metallic beam tube represents the classic example for the resistive wall instabilities. The permittivity in high-conductivity metals is in good approximation given by $\mu \simeq 1$ and $\varepsilon_{S} \approx-j \sigma / k$ leading to $\kappa \rightarrow \chi=\sqrt{j \mu \sigma k}$. The relevant equations are conveniently written in terms of the skin depth, $\delta=$ $\sqrt{2 / \mu \sigma k}$, and the Bessel function argument $\chi b=$ $\sqrt{2 j}(b / \delta)$

Thus, both propagation models obtain the coupling impedance of a radially unbound metal tube from the same formula for the wall impedance,

$$
R(b)=\frac{\chi}{\sigma} \frac{K_{0}(\chi b)}{K_{1}(\chi b)}
$$

and at high frequencies where $b \gg \delta$,

$$
R_{\infty}(b) \rightarrow(1+j) \sqrt{\mu k / 2 \sigma} .
$$

Applying these values to Eq. (13),

$$
Z_{\|}=\frac{R(b)}{2 \pi b}\left(\frac{1}{1+j k b R(b) / 2}\right)
$$

yields the resistive wall impedances per unit length, which is equal to the total coupling impedance in the assumed ultrarelativistic limit. Figure 2 shows the computed results for an "infinitely thick" aluminum beam tube with $\sigma=$ $3.5 \times 10^{7} / \Omega \mathrm{m}$ and a $b=5 \mathrm{~mm}$ radius. The skin depth 


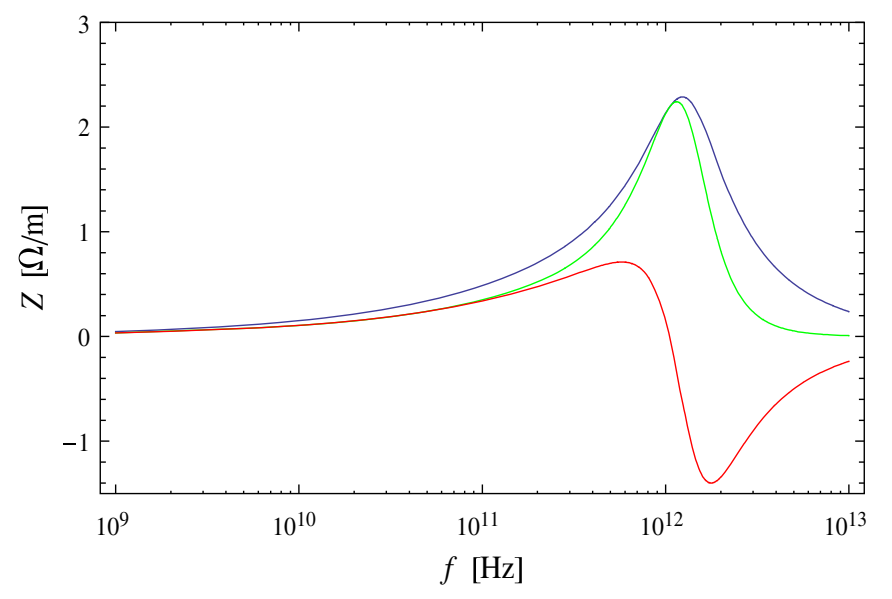

FIG. 2. Longitudinal coupling impedance per unit length of a radially unbound aluminum beam tube with $\sigma=3.5 \times$ $10^{7} / \Omega \mathrm{m}$ and a $b=5 \mathrm{~cm}$ radius.

equals the beam tube radius at $f \approx 3 \mathrm{~Hz}$ and the approximation $R_{\infty}(b)$ is indistinguishable, $<1 \%$, from the full expression in Eq. (51) above $\sim 1 \mathrm{kHz}$. It is noted that the present result agrees with Chao's Fig. (2.11) in Ref. [25].

\section{B. Metallic beam tube with finite thickness}

An important topic in the impedance analysis of a multilayer beam tubes is the treatment of the outermost layer, especially if it is vacuum. This topic can best be explored on a metal tube with finite thickness. As mentioned above, the difference in the two propagation models is apparent, primarily at low frequencies. In this section the longitudinal impedance of a single layer beam tube is considered, but the formalism applies equally to the complete matrix of multilayer structures. Considering "infinitely" long structures and accepting the description of the fields by modified Bessel functions within the material, any differences in the results must be attributed to the choice of the vacuum impedance at the outer layer.

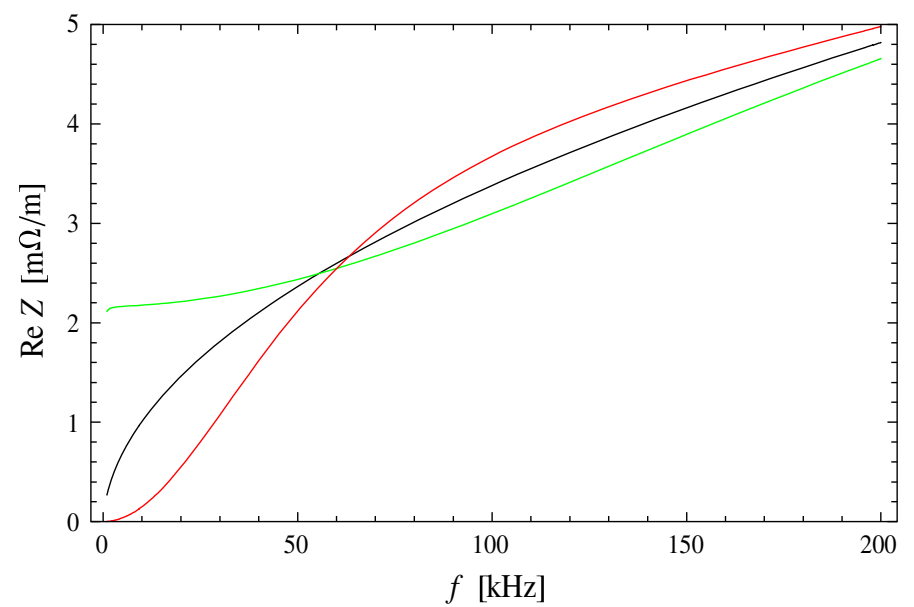

The longitudinal coupling impedance at "low" frequencies is found from Eq. (41),

$$
Z_{\|} \approx \frac{1}{2 \pi b} \frac{m_{e e}\left(b, r_{o}\right) R_{z}\left(r_{o}\right)-m_{e h}\left(b, r_{o}\right)}{m_{h h}\left(b, r_{o}\right)-m_{h e}\left(b, r_{o}\right) R_{z}\left(r_{o}\right)},
$$

with the matrix elements given by Eq. (27), together with $\varepsilon_{S} \approx-j \sigma / k$ and $\kappa$ replaced by $\chi=\sqrt{j \mu \sigma k}$. The wave impedance of vacuum at $r_{o}$ was found above to be in the ultrarelativistic case

$$
\begin{aligned}
& R_{z}\left(r_{o}\right)=0 \text { for the axial model and } \\
& R_{z}\left(r_{o}\right)=j H_{0}^{(2)}\left(k r_{o}\right) / H_{1}^{(2)}\left(k r_{0}\right) \text { for the radial model. }
\end{aligned}
$$

A first prototypical example is found in the resistive wall impedance of the standard straight stainless-steel tube for the extensively studied and well-documented PETRA III storage ring [16]. The parameters of the circular vacuum chamber are given as $47 \mathrm{~mm}$ aperture, $2 \mathrm{~mm}$ wall thickness, $1.5 \times 10^{6} / \Omega \mathrm{m}$ stainless-steel conductivity, and $\varepsilon$, $\mu \simeq 1$, implying a DC impedance of $\sim 2.4 \times 10^{-3} \Omega / \mathrm{m}$. The skin depth here equals the wall thickness at a frequency of $\sim 42 \mathrm{kHz}$, below which the exact solution expectedly will differ from the thick wall impedance approximation in Eq. (52).

Figure 3 depicts the numerical results in the ultrarelativistic limit that were obtained via the Wolfram MATHEMATICA program using only the full Bessel function expressions. The outermost vacuum layer was treated with the axial model (red), with the radial model (green), and for reference as an unbound tube (black). The results depend strongly on the impedance of the different terminations taken for vacuum, but do not allow a choice without experimental confirmation. The presence of a nearly flat resistive value below the critical skin depth frequency of $\sim 42 \mathrm{kH} z$ gives credence to the radial propagation model.

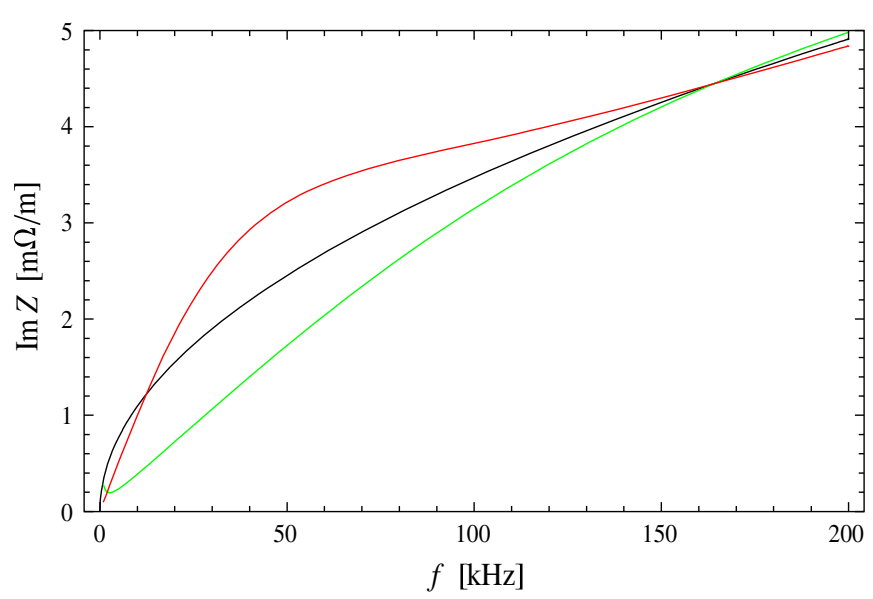

FIG. 3. Longitudinal impedance per unit length of a $2 \mathrm{~mm}$ thick thickness stainless-steel beam tube with $\sigma=1.5 \times 10^{6} / \Omega \mathrm{m}$ and a $b=23.5 \mathrm{~mm}$ radius [16], in red, green, and black, respectively, for the axial, radial, and unbound model. 
A second example is found in the LHC graphite collimator treated as a circular tube with an inner radius $b=$ $2 \mathrm{~mm}$, an outer radius $r_{o}=2.5 \mathrm{~cm}$, and the graphite conductivity $\sigma=7 \times 10^{4} / \Omega \mathrm{m}[19,24]$. The skin depth equals the wall thickness of $2.3 \mathrm{~cm}$ at about $7 \mathrm{kHz}$. The longitudinal impedance of the collimator is illustrated in Fig. 4 at very low frequencies in a linear scale, together with $Z / n$ in a log-log scale, at frequencies up to $1 \mathrm{GHz}$, where $Z$ is normalized to $n=f / f_{\text {rev }}=f / 11245 \mathrm{~Hz}$. The curves are shown in color to identify the model for the terminating vacuum layer used with red for the axial model and green for the radial model. Also shown are the impedance curves for the unbound tube from the exact formula, Eq. (51) (black), and the classic approximation with $R_{z}(b)=\chi / \sigma$ (dashed black). It is noted that in the lowfrequency range, at or below the frequency when the skin depth exceeds the wall thickness, the results and, in particular, their real part differ significantly from the classic approximation. In contrast to the axial model, the radial model provides the expected DC resistance. The results for the PETRA III beam tube and the LHC uncoated collimator exhibit similar low-frequency behavior, although the results below $1 \mathrm{kHz}$ are problematic. Notwithstanding the
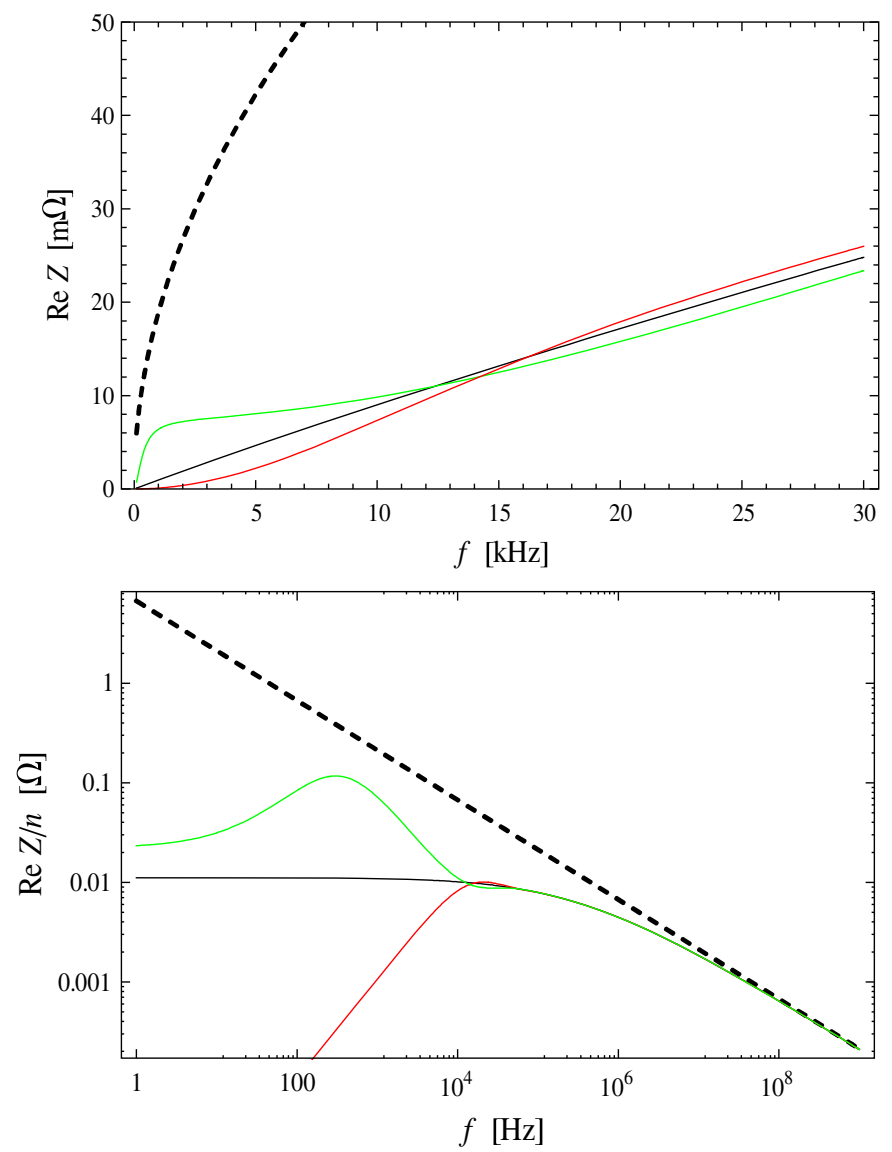

recent collimator paper [20], there is as yet not enough experimental data available to select either model.

\section{ULTRARELATIVISTIC TRANSVERSE IMPEDANCE}

The foregoing study of the longitudinal coupling impedance found no decisive difference between the axial and radial models at frequencies where the wall thickness exceeds the skin depth. A more definite statement would require comparing the transverse impedance from both models with experimental data in all frequency ranges. The transverse-wall impedance is given by the general expression, Eq. (41), independent of the model, and any "free coefficient" constraint is avoided by the exclusive use of TM mode propagation. However, its use for a broad study goes beyond the scope of this paper, where the demonstration of the matrix method is the primary goal.

The application of the matrix method is here demonstrated with the axial model for the analysis of transverse impedances in the ultrarelativistic limit where the expressions for the wall impedance are particularly simple. Although, in principle, the matrix method can be used
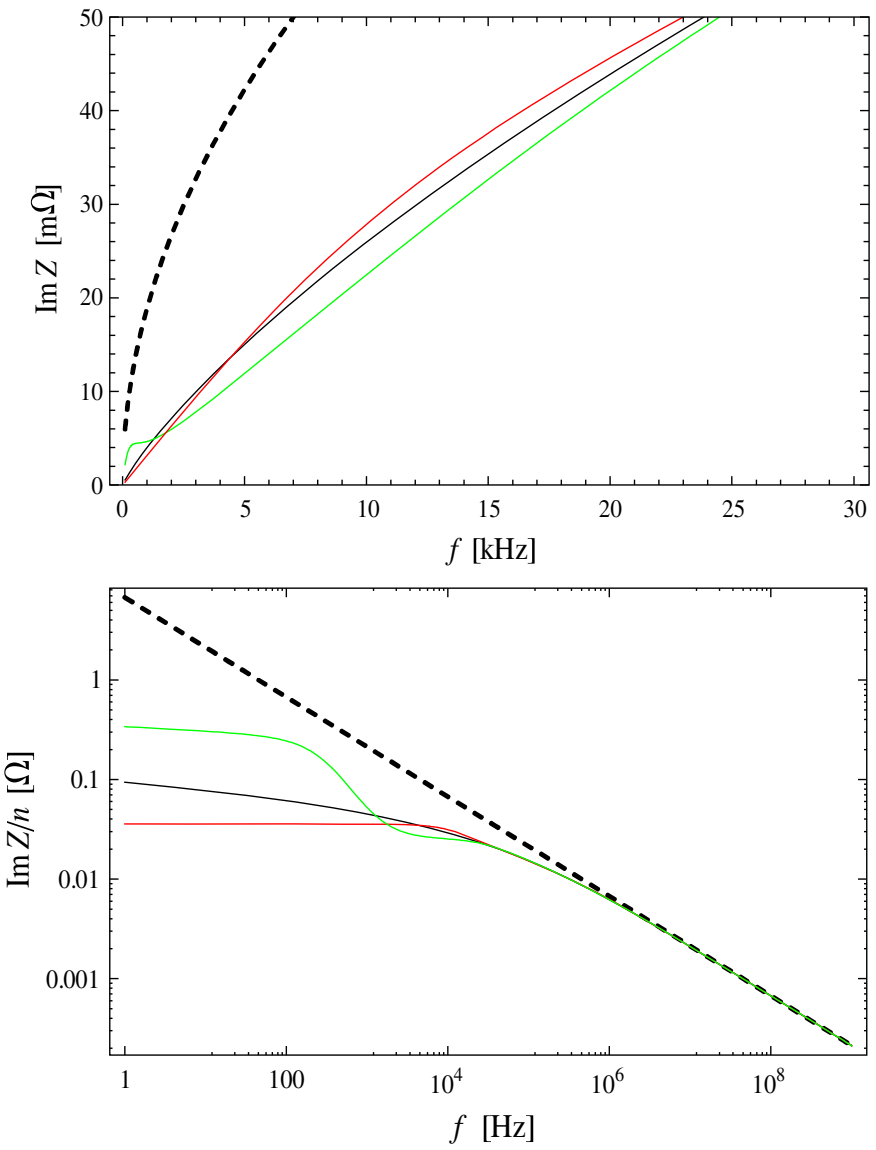

FIG. 4. Longitudinal coupling impedance of the LHC graphite collimator, $1 \mathrm{~m}$ long with radius $b=2 \mathrm{~mm}$ and conductivity $\sigma=$ $7 \times 10^{4} / \Omega \mathrm{m}$. The data are shown for the axial and radial model, respectively, in red and green. Also shown are the curves for the unbound thick layer from the exact (black) and from the approximate (dashed black) expressions. 
for finite beam energies by retaining a finite $\beta$ in the matrix elements, the formula for the wall impedance reduces in the ultrarelativistic limit to the ratio of two overall matrix elements,

$$
R_{z}(b) \approx-\frac{M_{\Theta e h}}{M_{\Theta h h}},
$$

which is to be entered into the expression for the transverse coupling impedance,

$$
Z_{T} \approx \frac{2}{\pi k b^{3}} R_{z}(b) /\left(1+j \frac{k b}{2} R_{z}(b)\right) .
$$

The matrix method does not depend on the number of layers, and for computational simplicity it is here demonstrated first on the LHC uncoated collimator but then it is extended to the two layer case of the $\mathrm{Cu}$ coated version. The simplifications gained become fully apparent when used for multilayer structures in connection with the Wolfram MATHEMATICA program. All computations in this section were performed with the full Bessel functions.

\section{A. Metallic single layer beam tube}

A first reference case for the transverse coupling impedance is found in the uncoated LHC graphite collimator. The collimator is considered as a metal tube, the parameters of which already are given above for the longitudinal impedance analysis. If treated as a single layer radially bound, the wall impedance in the axial model is obtained by using $R_{z}\left(r_{o}\right)=0$ in Eq. (50) leading to Eq. (56) with the matrix elements for finite size tubes in Eq. (35). The uncoated collimator, if treated as a radially unbound tube of uniform material, has the wall impedance

$$
R_{z}(b)=-\frac{\chi}{\sigma} \frac{K_{1}(\chi b)}{K_{1}^{\prime}(\chi b)}
$$

The transverse coupling impedance values of the uncoated collimator follow from Eq. (57). Figure 5 shows the real and imaginary transverse impedances, respectively, of the finite size single layer uncoated collimator as dashed green and red curves, together with those for the unbound layer in black. Also included is the absolute value for the classic "thick" wall impedance value, dashed black, from $R_{z}(b)=\chi / \sigma$. Not surprisingly, the full expression is required in the low-frequency region where $b<\delta$. Note that the real part of the transverse coupling impedance at low frequencies is lowered by the finite size of the layer.

\section{B. Double-layer beam tube}

The well-documented coated LHC graphite collimator affords an instructive example of multilayer beam tubes [19]. The $5 \mu \mathrm{m}$ thick $\mathrm{Cu}$ coating has a conductivity of $\sim 6 \times 10^{7} / \Omega \mathrm{m}$, and the skin depth equals the coating thickness at $\sim 170 \mathrm{MHz}$. The $2 \times 2$ matrix defining a multilayer beam tube is obtained by multiplying the individual matrices for each layer according to Eq. (23).



FIG. 5. Transverse coupling impedance in the ultrarelativistic limit for the $1 \mathrm{~m}$ long LHC graphite collimator, having inner and outer radii $b=2 \mathrm{~mm}$ and $r_{o}=25 \mathrm{~mm}$, and the conductivity $\sigma=7 \times 10^{4} / \Omega \mathrm{m}$. The real and imaginary values are shown, respectively, as dashed green and red curves. Also shown are the impedances for an unbound graphite collimator (black) and the absolute value of the classic thick wall approximation (dashed black).

Equation (35) gives the individual matrix elements for the transverse impedance to be used, although the hyperbolic approximations are not recommended in the lowfrequency range of interest here.

The numerical results for the transverse coupling impedance of the coated collimator are shown in Fig. 6, together with those for the uncoated collimator in order to highlight the impedance changes due to the coating. The real (green) and imaginary (red) parts of the impedances are illustrated for the coated unit by the solid and for the uncoated collimator by the dashed curves. Also plotted is the absolute impedance value for the classic thick wall



FIG. 6. Transverse coupling impedance of the $1 \mathrm{~m}$ long LHC coated graphite collimator, coated with a $5 \mu \mathrm{m}$ copper layer of $\sim 6 \times 10^{7} / \Omega \mathrm{m}$ conductivity (see Fig. 5). The real and imaginary impedance of the coated (uncoated) tube is shown by solid (dashed) green and red curves. Also shown are the absolute values of the impedances in the classic thick wall approximation for an unbound graphite (black) and copper (blue) collimator. 
approximations, with dashed black for an uncoated ceramic collimator, and blue for a copper collimator. The present numerical results are consistent with those recently published by the CERN team [20], but a detailed comparison with the experimental data for very low frequencies, which would involve extending to the radial model, is beyond the scope of this paper.

\section{CONCLUSION}

The matrix method presented in this paper provides a novel analysis of the longitudinal and transverse coupling impedances of multilayer circular cylindrical beam tubes. In order to apply the matrix method, each beam tube layer is defined by a $2 \times 2$ matrix relating the tangential field components of the TM wave propagating between the inner and the outer boundary. The beam tube fields can be defined in terms of the standard axial, or tentatively by a purely radial wave propagation model, and the matrix method can be applied in either case to find a result that is exact within the definition of the model. The matrix for the assembled beam tube is obtained by sequential matrix multiplication, thereby providing the wall impedance at the innermost radius by impedance mapping of the outermost impedance. Replacing the standard sequential field matching with matrix multiplications entails considerable analytical simplifications, and is well suited for computational work with the Wolfram MATHEMATICA program. For example, the wall impedance in the ultrarelativistic limit is given in the axial model by the ratio of only two matrix elements. As an illustration of the matrix method, the results for the longitudinal and transverse impedance of a PETRA III stainless-steel beam tube and the LHC coated graphite collimator are computed and graphically shown.

\section{ACKNOWLEDGMENTS}

This work was supported by Brookhaven Science Associates, LLC, under Contract No. DE-AC0298CH10886 with the U.S. DOE.

[1] K. Neil and A. Sessler, Rev. Sci. Instrum. 36, 429 (1965).

[2] J. Laslett, K. Neil, and A. Sessler, Rev. Sci. Instrum. 36, 436 (1965).

[3] B. Zotter, Part. Accel. 1, 311 (1970).

[4] B. W. Zotter and S. A. Kheifets, Impedances and Wakes in High-Energy Particle Accelerators (World Scientific, Singapore, 1997), p. 157.

[5] H. Hahn and A. Ratti, RHIC Project Report No. AD/ RHIC/RD-106, BNL, 1996.

[6] G. R. Lambertson, Reports No. LBNL-44454 and CBP Note 326, 1999.

[7] L. Vos, CERN Report No. CERN-AB-2003-093 (ABP).

[8] M. Ivanyan, E. Laziev, V. Tsakanov, A. Vardanyan, S. Heifets, and A. Tsakanian, Phys. Rev. ST Accel. Beams 11, 084001 (2008).
[9] N. Marcuvitz, in Radial Transmission Lines, edited by C. G. Montgomery, R.H. Dicke, and E.M. Purcell, Principles of Microwave Circuits (McGraw-Hill Book Co., New York, 1948), p. 240.

[10] V. Danilov, S. Henderson, A. Burov, and V. Lebedev, in Proceedings of the 8th European Particle Accelerator Conference, Paris, 2002 (EPS-IGA and CERN, Geneva, 2002), p. 1464.

[11] H. Hahn, Reports No. C-A/AP \#336 and BNL-818672008-IR, BNL, 2008.

[12] H. Hahn, in Proceedings of the 23rd Particle Accelerator Conference, 2009, Vancouver, BC, poster TH5PEP082, http://trshare.triumf.ca/ pac09proc/Proceedings/.

[13] R.L. Gluckstern, Analytic Methods for Calculating Coupling Impedances, Particle Accelerator School, Phoenix, AZ, 2000; Yellow Report CERN 2000-011, 2000.

[14] A. M. Al-Khateeb, R. W. Hasse, O. Boine-Frankenheim, W. M. Daqa, and I. Hofmann, Phys. Rev. ST Accel. Beams 10, 064401 (2007).

[15] A. Burov and V. Lebedev, in Proceedings of the 8th European Particle Accelerator Conference, Paris, 2002 (Ref. [10]), p. 1452.

[16] M. Ivanyan, E. Laziev, V. Tsakanov, A. Vardanyan, A. Tsakanian, and R. Wanzenberg, PETRA III Storage Ring Resistive Wall Impedance, in ICFA Beam Dynamics Newsletter, edited by R. Wanzenberg, 2008, http://icfausa.jlab.org/archive/newsletter.shtml, p. 125.

[17] F. Caspers, G. Dome, C. Gonzalez, E. Jensen, E. Keil, M. Morvillo, F. Ruggiero, G. Schröder, B. Zotter, and M. D'Yachkov, in Proceedings of the 18th Particle Accelerator Conference, New York, 1999 (IEEE, New York, 1999), p. 1408.

[18] A. Mostacci, F. Caspers, L. Vos, and U. Iriso, in Proceedings of the 20th Particle Accelerator Conference, Portland, OR, 2003 (IEEE, New York, 2003), p. 1801.

[19] A. Koschik, F. Caspers, E. Métral, L. Vos, and B. Zotter, in Proceedings of the 9th European Particle Accelerator Conference, Lucerne, 2004 (EPS-AG, Lucerne, 2004), p. 1876.

[20] F. Roncarolo, F. Caspers, T. Kroyer, E. Métral, N. Mounet, B. Salvant, and B. Zotter, Phys. Rev. ST Accel. Beams 12, 084401 (2009).

[21] S. Krinsky, B. Podobedov, and R. L. Gluckstern, Phys. Rev. ST Accel. Beams 7, 114401 (2004).

[22] W. Panofsky and W. Wenzel, Rev. Sci. Instrum. 27, 967 (1956).

[23] J.A. Stratton, Electromagnetic Theory (McGraw-Hill Publishing Co., New York, 1941), Chap. VI, p. 354.

[24] E. Métral, B. Zotter, and B. Salvant, in Proceedings of the 2007 Particle Accelerator Conference, Albuquerque, New Mexico, 2007, http://www.jacow.org/, p. 4216.

[25] A. W. Chao, Physics of Collective Beam Instabilities in High Energy Accelerators (John Wiley \& Sons, New York, 1998).

[26] W. R. Smythe, Static and Dynamic Electricity (McGrawHill Book Co., New York, 1968), p. 371.

[27] S. Silver, Microwave Antenna Theory and Design (Boston Technical Publishers, Boston, MA, 1964), p. 75. 